\title{
Measurements of Electric Performance and Impedance of a 75 Ah NMC Lithium Battery Module
}

\section{Jensen, Søren Højgaard; Engelbrecht, Kurt}

Published in:

Journal of The Electrochemical Society

Link to article, DOI:

10.1149/2.088206jes

Publication date:

2012

Document Version

Publisher's PDF, also known as Version of record

Link back to DTU Orbit

Citation (APA):

Jensen, S. H., \& Engelbrecht, K. (2012). Measurements of Electric Performance and Impedance of a 75 Ah NMC Lithium Battery Module. Journal of The Electrochemical Society, 159(6), A791-A797.

https://doi.org/10.1149/2.088206jes

\section{General rights}

Copyright and moral rights for the publications made accessible in the public portal are retained by the authors and/or other copyright owners and it is a condition of accessing publications that users recognise and abide by the legal requirements associated with these rights.

- Users may download and print one copy of any publication from the public portal for the purpose of private study or research.

- You may not further distribute the material or use it for any profit-making activity or commercial gain

- You may freely distribute the URL identifying the publication in the public portal 


\title{
Measurements of Electric Performance and Impedance of a $75 \mathrm{Ah}$ NMC Lithium Battery Module
} Søren Højgaard Jensen,, ${ }^{*, z}$ Kurt Engelbrecht, and Carlos Bernuy-Lopez

Department of Energy Conversion and Storage, Technical University of Denmark, Ris $\phi$ Campus, 4000 Roskilde, Denmark

\begin{abstract}
Detailed characterization of battery modules is necessary to construct reliable models that incorporate performance related aspects of the modules such as thermodynamics, electrochemical reaction kinetics and degradation mechanisms. Charge-discharge curves, temperature and battery impedance measurements can provide information about these aspects. Charge-discharge curves can be used to measure the battery open circuit voltage and the internal resistance. Temperature measurements provide information about the thermodynamic reactions and impedance spectra yield detailed information about the reaction kinetics. In this paper we present the measurement methods used to examine the internal resistance, the capacity and the impedance of a 75 Ah NMC battery module. In order to measure the impedance of the battery module and of the individual cells in the module, we combine the single sine technique and the Laplace transformed excitation signal technique which each have pros and cons. By combining the two impedance measurement techniques we are able to reduce the measurement time by a factor of 20 relative to ordinary single-sine measurements. Further we use the impedance measurements to calculate the overvoltage as a function of state of charge and the difference between charging overvoltage and discharging overvoltage and compare it with measurements.

(C) 2012 The Electrochemical Society. [DOI: 10.1149/2.088206jes] All rights reserved.
\end{abstract}

Manuscript submitted December 9, 2011; revised manuscript received March 20, 2012. Published April 6, 2012.

During the last decade a rapidly increasing interest in batteries for propulsion in the transport sector has emerged. Since battery performance is drastically affected by the operation pattern it is important to characterize the battery degradation as a function of the operation pattern. This is not a trivial task since the battery performance is not a measurable quantity in itself, but covers several aspects such as the internal resistance and the capacity. Further, the operation pattern covers several aspects such as the Depth of Discharge (DOD), State of Charge (SOC), C-rate, temperature and calendar life.

The battery module wear due to specific operation patterns can be analyzed with micro-cycle tests where a single operating parameter such as the battery temperature is varied slightly while keeping the other operating parameters as constant as possible, e.g. the chargedischarge cycles are kept constant. The internal resistance, the capacity and the impedance of the cells and the module can be measured before and after each series of micro-cycles. In this way it is possible to map the battery module wear as a function of the various operating conditions and thus to develop a model that predicts the module wear due to a more complex operating pattern, as described by Safari et al. ${ }^{1}$

Battery impedance spectra provide valuable knowledge about the reaction kinetics of physical/chemical processes taking place inside the battery. For this reason the impedance spectra can provide information that can be used to develop improved state of health ( $\mathrm{SOH})$ models and to describe how the battery voltage (or current) responds to changes in the current (or voltage). ${ }^{2,3}$

It is usually very time consuming to measure impedance spectra on batteries because some of the investigated electrochemical processes occur very slowly. This is in particular true for single-sine methods where the impedance is measured at single frequency at a time. With time domain methods (TDM) it is possible to simultaneously measure the impedance at all frequencies within the measurement range. The minimum frequency that accurately can be analyzed by a TDM measurement is the inverse of the measurement time. ${ }^{4}$ At high frequencies the measurement accuracy of TDM is limited by the time resolution of the voltage or current perturbation and the time resolution and accuracy of the measurement of the resulting current or voltage response from the battery. Normally, this limits the maximum frequency that can be analyzed by TDM below $100 \mathrm{~Hz}$. For this reason it is interesting to combine high frequency single-sine measurements with low frequency TDM measurements to conduct fast and accurate impedance measurements in a broad frequency range.

*Electrochemical Society Active Member.

${ }^{\mathrm{z}}$ E-maiL: shjj@risoe.dtu.dk
It should be noted that multi-sine techniques exist ${ }^{5}$ which can decrease the measurement time by approximately a factor of 4 compared to single-sine measurements. ${ }^{6}$ Further, real-parallel impedance solutions like the 'PAD4' by Zahner can measure impedance on several cells simultaneously. Unfortunately, the equipment for both multisine and real-parallel techniques is generally more expensive than the equipment used for the presented measurement technique.

Several techniques to convert TDM measurements into frequency domain measurements (FDM) exist. ${ }^{4,7,8}$ Klots et al. have recently presented a work on combined TDM and EIS measurements of a Li-ion cell with an internal resistance on the order of $0.5 \Omega{ }^{9}$ Here we present results on combined TDM and EIS measurements, but we use the TDM method to simultaneously obtain impedance spectra on eight cells in a battery module, and the cell impedances are on the order of $1 \mathrm{~m} \Omega$.

The presented transformation of the TDM measurements into the frequency domain uses an equivalent circuit based on 4 (RC) circuits in series with a resistor and a capacitor, where (RC) is a parallel connection between a resistor and a capacitor. The equivalent circuit is used to model the overvoltage as a function of time due to a step current of 1.0 Ampere, and the values of the resistors and capacitors obtained from the modeling are used to calculate the impedance. The number of (RC) circuits was chosen to be the smallest number that still allowed an accurate fit of the overvoltage.

Finally, we use the measured impedance to calculate the overvoltage as a function of SOC due to a complex charge discharge cycle and compare it with the measured overvoltage.

\section{Experimental}

The tested battery module is a SLPB125255255H_8S1P module supplied by Kokam Co. Ltd. The module consists of eight serially connected 75 Ah cells with lithium-nickel-manganese-cobalt-oxide cathodes and lithium-carbon anodes. The cell electrolyte consist of lithium salt e.g. LiPF6, organic solvent such as ethylene carbonate, gel polymer and performance/safety enhancing additives. The cells are usually referred to as NMC cells and Kokam supplies the following technical specification for the NMC cells: The minimum discharge cell voltage is $3.0 \mathrm{~V}$, the nominal voltage is $3.7 \mathrm{~V}$ and the maximum charge voltage is $4.15 \pm 0.03 \mathrm{~V}$. The module is $29.3 \mathrm{~cm}$ high, $27,5 \mathrm{~cm}$ wide and $10.5 \mathrm{~cm}$ long.

The battery module voltage, the individual cell voltages, the current and the module temperature were consecutively logged with a Keithley 2750 system with two Keithley 7700 cards and a Keithley 7702 card. A thermistor placed between cell 1 and cell 2 was used to measure 


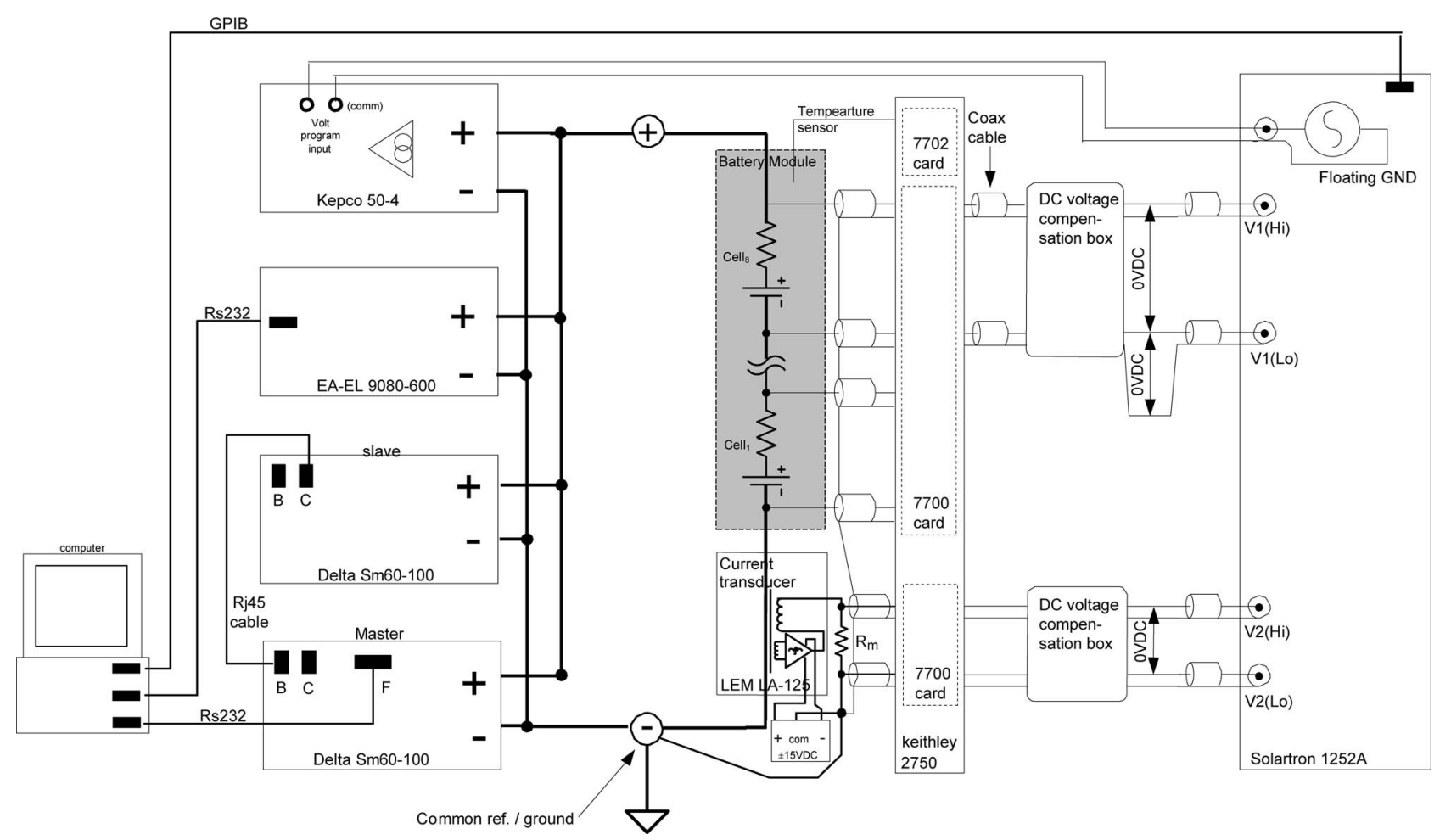

Figure 1. Diagram of the wiring between the most important parts of the test system: Power supplies: Delta SM60-100, E-load: EA-EL 9080-600, Current sensor: LEM LA-125 with measurement resistor $\mathrm{R}_{\mathrm{m}}$, Frequency analyser: Solartron 1252A, Multiplexer: Keithley 2750 and the Battery module: Kokam SLPB125255255H_8S1P.

the temperature inside the module. The thermistor was placed $1.5 \mathrm{~cm}$ below the top and $10 \mathrm{~cm}$ from one of the sides of the module.

The module was charged with two Power supplies DeltaElektronika SM 60-100 in parallel and discharged with an E-load EA9080-600. The Power supplies and E-load could be de-coupled from the main electric circuit with power relays (not shown in Figure 1).

The module and cell impedance was measured with normal singlesine impedance spectroscopy using a Solartron 1252A. A Kepco BOP 50-4M was used to boost the $16 \mathrm{~mA}$ AC current from the Solartron to 1 Ampere AC. When current passes the current transducer LEM LA-125 it results in current passing through the measurement resistor $\mathrm{R}_{\mathrm{m}}$ and accordingly a voltage drop across $\mathrm{R}_{\mathrm{m}}$. Dividing this voltage drop with the current passing through the current transducer the "resistance" of the combined current transducer and $\mathrm{R}_{\mathrm{m}}$ is obtained. Using an $\mathrm{AC}$ current instead of a DC current, we can measure the impedance of the combined current sensor and measurement resistor. We call this impedance $Z_{L E M}$. During the impedance measurements the AC voltage drop across $\mathrm{R}_{\mathrm{m}}$ was measured with "V2" on the Solartron 1252A.

The current passing through the battery module changes the voltage of the individual cells and of the module as a whole. The cell voltages or the module voltage was measured with "V1" on the frequency analyzer. The coax-cables from the Solartron to the individual cellelectrodes were multiplexed with a Keithley 2750 and two Keithley 7700 cards to enable automated measurements without needing to move the impedance cables.

Prior to the impedance measurements the DC voltage compensation boxes were automatically adjusted so that the DC voltage from the module or one of the cells was removed. This means that only the AC voltage is transferred to the frequency analyzer in order to use the $5 \mathrm{~V}$ common-mode rejection of the Solartron 1252A and to make use of the most sensitive measurement range of the frequency analyzer.

V1/V2 was recorded with the Solartron 1252A in the frequency range $2.6 \mathrm{kHz}$ to $0.6 \mathrm{mHz}$. The impedance of the battery was obtained from the equation

$$
Z_{\text {Bat }}=\frac{\mathrm{V} 1}{\mathrm{~V} 2} \cdot Z_{L E M}
$$

At high AC frequencies the power supplies: Delta SM60-100 and the E-load: EA-EL 9080-600 stop acting as perfect galvanostats and significant error currents pass through these devices. However, the current transducer is in series with the battery module so it only senses the current passing the battery module. The $\mathrm{AC}$ voltage generated in the battery module and its cells is caused by the AC current applied to the battery. Thus the impedance measurement is not affected by the leak currents in the E-load and power supplies.

The module and cell impedances were also measured with a Laplace transform of the module and cell over-voltage. The overvoltages were measured as a function of time after onset of a stepcurrent of 1 Ampere. The data acquisition lasted one hour and the data was measured every $2 \mathrm{~s}$. The Kepco BOP 50-4M was used to apply the step-current for the Laplace measurements.

The module charge capacity was tested with a charge-discharge curve ranging from the manufacturer's maximum charge voltage to the minimum discharge voltage. We define the module to be fully charged when the voltage of one of the cells in the battery module reach $4.15 \mathrm{~V}$ at $0.13 \mathrm{C}$ which corresponds to a charge current of $-10 \mathrm{~A}$. The state of charge (SOC) of the fully charged battery module is defined as $100 \%$ SOC. The fully discharged battery module, i.e. when one of the cells in the battery module reaches $3.0 \mathrm{~V}$ at $0.13 \mathrm{C}$ i.e. at a discharge charge current of $10 \mathrm{~A}$, is defined as $0 \%$ SOC.

SOCs between $0 \%$ and $100 \%$ are measured by Coulomb counting i.e. by subtracting the net charge flow from the fully charged module divided with the measured module charge capacity.

The module OCV is measured as a function of SOC by a method previously described by Shark and Doerffel who used it for fast OCV measurements at high C-rates. ${ }^{10}$ Here we measure the OCV vs SOC from a charge-discharge cycle which consists of a series of discharge steps from $100 \%$ SOC to $0 \%$ SOC followed by a series of charge steps 


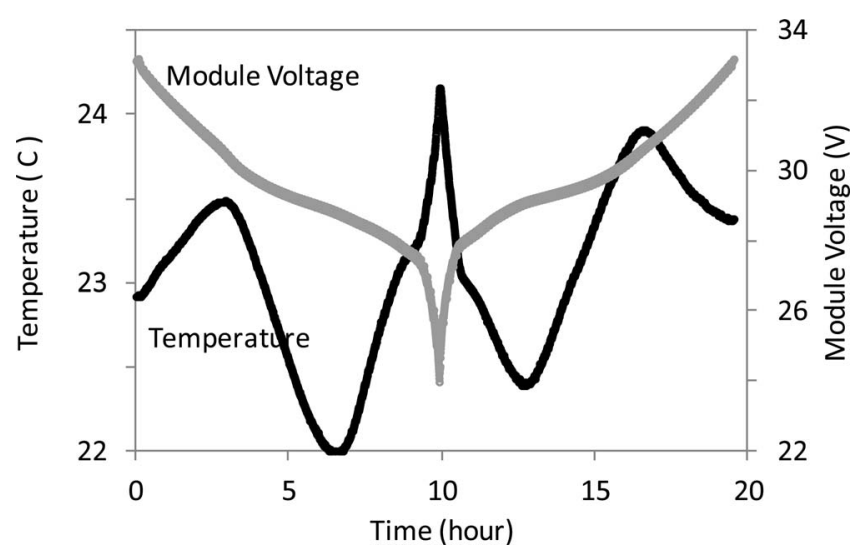

Figure 2. Voltage and temperature measured as a function of time during a charge-discharge cycle of a 75 Ah NMC battery module.

from $0 \%$ SOC to $100 \%$ SOC. Each step lasts six minutes and consists of five minutes of either charging or discharging at $0.13 \mathrm{C}$, i.e. at either $-10 \mathrm{~A}$ or $10 \mathrm{~A}$, and one minute at $0 \mathrm{C}$, i.e. at $0 \mathrm{~A}$. The voltage measurement during the $0 \mathrm{C}$ periods in the charge steps and discharge steps is used to determine the OCV vs. SOC. This is described in further detail with the presentation of the measurements in the result section.

Non-ideal Coulumbic efficiency can lead to large accumulated errors for this type of SOC measurements if the battery is cycled several times without reaching fully charged or discharged condition where re-calibration of the SOC is possible. Here we present a chargedischarge curve from $100 \%$ SOC to $0 \%$ SOC and back to $100 \%$ SOC. This SOC definition does not fully account for self-discharge with time. However, the self-discharge of the module prior to any degradation tests was approximately $1 \mathrm{mV}$ per day and can thus be ignored during the presented charge-discharge curves which lasted for approximately 20 hours.

\section{Results}

The battery module temperature and voltage were measured as a function of time during a full charge-discharge cycle, see Figure 2. Before the charge-discharge cycle, the module was charged to $100 \%$ SOC. This was done in steps of 6 minutes. In each step, the battery was first charged with -10 A for five minutes, and the current was reduced to $0 \mathrm{~A}$ for one minute. As soon as one of the eight cells in the module reached the maximum charge voltage of $4.15 \mathrm{~V}$, the current was interrupted and the current state of charge was defined as $100 \%$ SOC.

Immediately after this, the module was discharged with $10 \mathrm{~A}$ for 5 minutes. Then the battery current was set to $0 \mathrm{~A}$ for one minute. This was repeated until one of the eight cells in the module reached $3 \mathrm{~V}$. After this, the module was charged in steps of 5 minutes with $-10 \mathrm{~A}$ and 1 minute at $0 \mathrm{~A}$ until one of the eight cells reached $4.15 \mathrm{~V}(100 \%$ SOC).

The battery capacity was measured as the current multiplied with the total time during discharging. The battery capacity was found to be $75.3 \mathrm{Ah}$ when the ambient temperature was $22^{\circ} \mathrm{C}$. The temperature of the battery module at the beginning of the charge-discharge cycle is higher than the ambient temperature due to pre-charging to reach $100 \%$ SOC.

The module voltage as a function of state of charge is shown in Figure 3. The gray line shows the module voltage during the 5 minute-periods at $0.13 \mathrm{C}$. The black points in the figure show the module voltage just before the end of the 1 minute-periods at $0 \mathrm{C}$. As described by Abu-Sharkh and Doerffe ${ }^{10}$ an estimate of the OCV at a given SOC can be obtained as the average voltage of the upper and lower black points. If the periods at $0 \mathrm{C}$ had been sufficiently long,

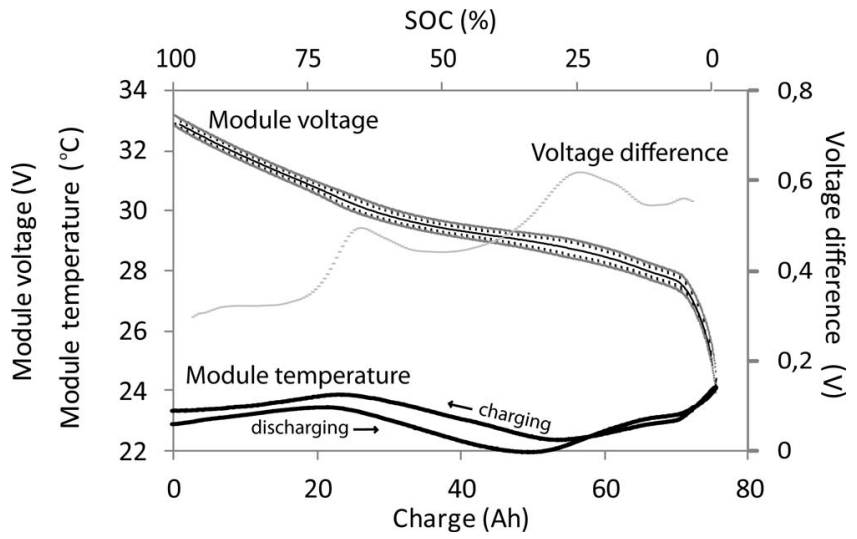

Figure 3. Charge-discharge curve for the $75 \mathrm{Ah}$ NMC battery module. The charge-discharge curve is made as a series of discharge steps followed by a series of charge steps. Each step takes 6 minutes: 5 minutes at $0.13 \mathrm{C}$ and one minute at $0 \mathrm{C}$. The gray line shows the module voltage during $0.13 \mathrm{C}$. The black points show the module voltage during $0 \mathrm{C}$. The thin black line shows the SOC calculated from the black points. The gray points show the voltage difference between the upper and lower gray line. The thick black line shows the module temperature.

the module voltage would relax until it reaches OCV. The thin black line in Figure 3 show this calculated OCV estimation. The difference between charging and discharging voltage as a function of SOC are shown with gray dots. The voltage was measured immediately before the one-minute current interruptions in the charge and discharge steps. In the figure, the module temperature is also shown as a function of state of charge with a thick black line.

The module impedance was measured at $90 \%$ SOC. This was done both in an ordinary single-sine measurement with a Solartron 1252A and with a Laplace transformed over-voltage curve. The over-voltage as a function of time after onset of a step-current of -1.0 Ampere is shown in Figure 4. The over-voltage is measured with a frequency of approximately $0.5 \mathrm{~Hz}$ for one hour.

The criteria for the model of the overvoltage in Figure 4 are threefold. Firstly, the model should fit the data in the time domain as well as possible. From the inset in Figure 4, which shows the residual between the measurement data and the model data, it is seen that this is indeed fulfilled by the chosen model. Secondly, it should be possible to

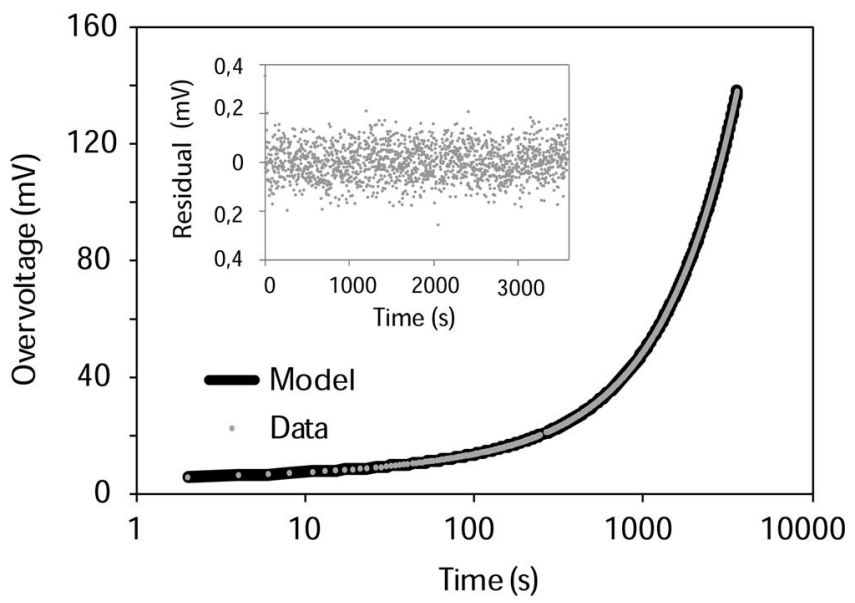

Figure 4. Overvoltage measured on a $75 \mathrm{Ah} \mathrm{NMC} \mathrm{battery} \mathrm{module} \mathrm{as} \mathrm{a} \mathrm{function}$ of time after onset of -1.0 A current. The gray dots are the measured data. The black line is a model of the overvoltage. The inset shows the residual difference between the model and the measured data. We use the model to derive the low frequency impedance of the battery module. 
analytically transform the model into the frequency domain. Thirdly the model should provide impedance data that is in good agreement with the single-sine measurements. We do not assign a physical meaning to the individual variables in the model of the overvoltage. However, the sum of resistances in the model is described as the internal resistance of the battery module as presented in the discussion section.

The model of the overvoltage is obtained from an equivalent circuit, $\mathrm{R}_{\mathrm{s}} \mathrm{C}_{\text {bat }}(\mathrm{RC})_{1}(\mathrm{RC})_{2}(\mathrm{RC})_{3}(\mathrm{RC})_{4}$, where $\mathrm{R}$ is a resistor and $\mathrm{C}$ is a capacitor, $(\mathrm{RC})$ is a parallel connection between a resistor and a capacitor and $\mathrm{R}_{\mathrm{s}}, \mathrm{C}_{\mathrm{bat}}$ and $(\mathrm{RC})_{1-4}$ are serially connected. As shown in the discussion section, the number of (RC)-circuits was chosen as the smallest number that fulfilled the first of the above mentioned criteria.

The current in the time domain can be written as $I(t)=I_{0} \cdot u(t)$ where $I_{0}$ is the current amplitude, i.e. -1.0 Ampere, and $u(t)$ is a step function which is 0 when $t<0$ and 1 when $t \geq 0$. The Laplace transform of $I(t)$ is $I(s)=I_{0} / s$, where $s=j \omega, j$ is the complex unity and $\omega$ is the angular frequency. The overvoltage in the frequency domain (or s-domain), $U_{m}(s)$, is the product of $I(s)$ and the impedance of the equivalent circuit $Z_{m}(s)$, i.e.

$$
\begin{aligned}
U_{m}(s)= & I(s) \cdot Z_{m}(s)=\frac{I_{0}}{s} \cdot\left(R_{s}+\frac{1}{s C_{b a t}}+\frac{R_{1}}{1+s R_{1} C_{1}}\right. \\
& \left.+\frac{R_{2}}{1+s R_{2} C_{2}}+\frac{R_{3}}{1+s R_{3} C_{3}}+\frac{R_{4}}{1+s R_{4} C_{4}}\right)
\end{aligned}
$$

The overvoltage in the time domain, $U_{m}(t)$ is given as the inverse Laplace transform of $U_{m}(s)$ :

$$
\begin{aligned}
U_{m}(t)= & u(t) \cdot I_{0}\left[R_{s}+t \cdot C_{b a t}+R_{1}\left(1-e^{\frac{-t}{R_{1} C_{1}}}\right)+R_{2}\left(1-e^{\frac{-t}{R_{2} C_{2}}}\right)\right. \\
& \left.+R_{3}\left(1-e^{\frac{-t}{R_{3} C_{3}}}\right)+R_{4}\left(1-e^{\frac{-t}{R_{4} C_{4}}}\right)\right]
\end{aligned}
$$

Values were assigned to the variables in the expression of $U_{m}(t)$ by fitting the model of $U_{m}(t)$ to the data in Figure 4, i.e. by minimizing the error sum

$$
E r r=\sum_{t_{n}} \frac{1}{t_{n}}\left(U_{\mathrm{m}}\left(t_{n}\right)-U_{d}\left(t_{n}\right)\right)^{2}
$$

where $t_{n}$ is the time after onset of the step-current when the $\mathrm{n}^{\text {th }}$ measurement point was recorded. $U_{d}\left(t_{n}\right)$ are the data points shown in Figure 4.

A weighing factor of $1 / t_{n}$ was used in the calculation of Err to equalize the weighting of the involved variables as much as possible and thus stabilizing the conversion of the fitting routine: Suppose $(\mathrm{RC})_{1}$ primarily models the part of the impedance spectrum from $0.1 \mathrm{~Hz}$ to $0.01 \mathrm{~Hz}$ and $(\mathrm{RC})_{4}$ primarily models the part of the impedance spectrum from $0.001 \mathrm{~Hz}$ to $0.0001 \mathrm{~Hz}$. This means the $U_{m}(t)$ measurements from 10 seconds to 100 seconds are primarily modeled by $(\mathrm{RC})_{1}$ and the $U_{m}(t)$ measurements from 1000 seconds to 10000 seconds are primarily modeled by $(\mathrm{RC})_{4}$ (temporarily disregarding that the we only measured $U_{m}(t)$ for 3600 seconds). Because $U_{m}(t)$ was measured approximately every $2^{\text {nd }}$ second, 45 measurements were conducted from 10 to 100 seconds and 4500 measurements were obtained between 1000 and 10000 seconds. This means

$$
\sum_{t_{n}=10 s}^{100 s} \frac{1}{t_{n}} \approx \sum_{t_{n}=1000 s}^{10000 s} \frac{1}{t_{n}} \approx \frac{1}{2} \ln (10)
$$

which means the variables in $(\mathrm{RC})_{1}$ and $(\mathrm{RC})_{4}$ are approximately equally weighted when we minimize the expression in (4).

In order to stabilize the modeling routine we fix the value of $R_{s}$ using the following relation derived from equation 3 .

$$
\begin{aligned}
R_{s}= & \frac{U_{m}\left(t_{1}\right)}{I_{0}}-t_{1} \cdot C_{b a t}-R_{1}\left(1-e^{\frac{-t_{1}}{R_{1} C_{1}}}\right)-R_{2}\left(1-e^{\frac{-t_{1}}{R_{2} C_{2}}}\right) \\
& -R_{3}\left(1-e^{\frac{-t_{1}}{R_{3} C_{3}}}\right)-R_{4}\left(1-e^{\frac{-t_{1}}{R_{4} C_{4}}}\right)
\end{aligned}
$$

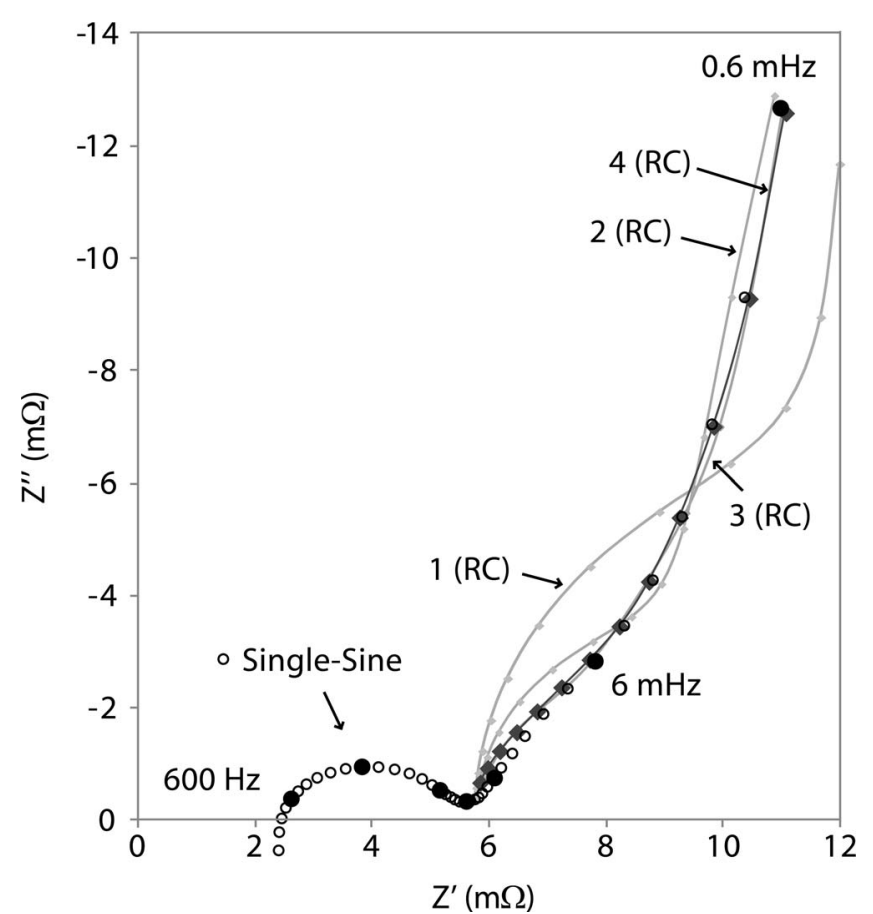

Figure 5. Impedance measured on the $75 \mathrm{Ah}$ NMC module. The Impedance is measured both with a Single-Sine method and a Laplace method (equation 3) with 1, 2, 3 and 4 (RC)s. The Single-Sine measurements shown with solid black markers denote the frequency decades.

where $U_{m}\left(t_{1}\right)$ is the overvoltage measured at $t_{1}$ and $t_{1}$ is the time after onset of the step-current where the first measurement of the overvoltage occur.

The values of the remaining variables $C_{b a t}, R_{1}, C_{1}, R_{2}, C_{2}, R_{3}, C_{3}$, $R_{4}$ and $C_{4}$ obtained from the fitting routine were used to calculate $Z_{m}(s)$, and the result is shown in Figure 5 (legend: 4 (RC)) together with the battery module impedance measured with the single-sine measurement method. From the figure it is seen that the two methods yields reasonably accurate data below $60 \mathrm{mHz}$. Above $60 \mathrm{mHz}$ the laplace method measurements (not shown) increasingly deviate from the single-sine measurements possibly due to the limited data acquisition frequency. The figure also shows $Z_{m}(s)$ obtained from modeling the overvoltage using an equivalent circuit having 1,2 and 3 (RC)s in series with a capacitor and a resistor as described in the discussion section.

In order to measure the changes in the electrode kinetics of the individual cells of the module, we measured impedance spectra on the individual cells in the battery module. Just as we did with the measurements of the battery module impedance spectra shown in Figure 5, the spectra were measured both with the single sine measurement method and with the TDM measurement method. Again, $R_{s}$ was fixed in order to assure a stable conversion of the fitting routine using the expression in (6). The obtained impedance data is shown in Figure 6.

\section{Discussion}

The first part of the discussion section examines the thermal behavior of the battery module presented in Figure 2 and Figure 3. The changes in the exothermal/endothermal behavior of the battery module is thought to be due to entropy changes arising from structural transformations in the Li-C anode and NMC cathode phase changes ${ }^{11-14}$ which in turn lead to the observed changes of the temperature inside the battery module. In Figure 7, this is exemplified by schematic data for two local minima of the battery temperature as observed in Figure 3. At $\mathrm{SOC}_{2}$ the entropy of the electrochemical processes in the battery changes sign. The heat power $P_{e}$ from the electrochemical 


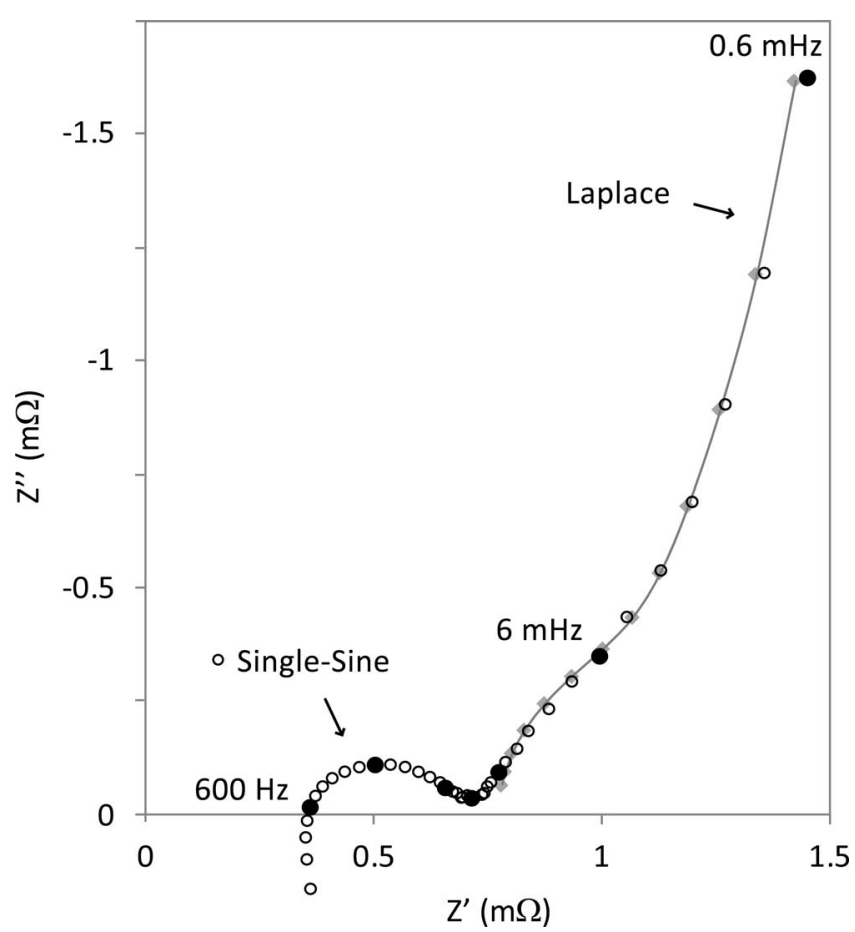

Figure 6. Impedance measured on a single cell in the $75 \mathrm{Ah}$ NMC module. The Impedance is measured both with a Single-Sine method and a Laplace method. The Single-Sine measurements shown with solid black markers denote the frequency decades. The two methods overlap reasonably well at frequencies below $60 \mathrm{mHz}$.

processes can be described as

$$
P_{e}=\frac{I}{n F} \cdot T \Delta S
$$

where $I$ is the current in Ampere, $n$ is the number of electrons involved in the electrochemical process, $F$ is faradays constant, $T$ is the temperature and $\Delta S$ is the entropy change. Note that $P_{e}$ changes sign with the sign of the current. The joule heat due to current passing through

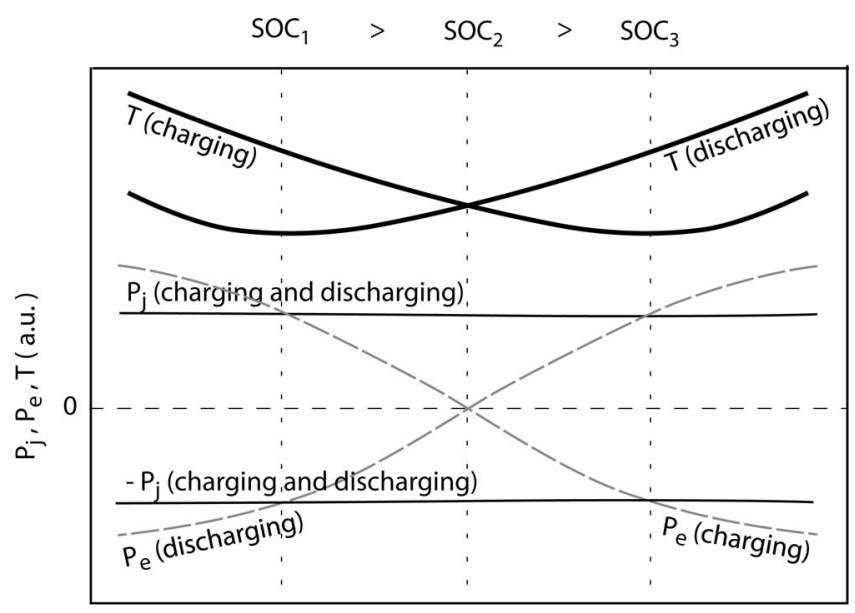

SOC (\%)

Figure 7. A description of the observed local minima in the battery temperature during charging and discharging shown in Figure 3 by joule heat and changes in the entropy of the electrochemical processes. The local maxima in the battery temperature can be described in a similar manner. the electrolyte is given as

$$
P_{J}=R_{i} I^{2}
$$

where $R_{i}$ is the electrolyte resistance. $P_{J}$ is positive during both charging and discharging. The change in the battery temperature per unit time can be described as

$$
\frac{d T}{d t}=\left(P_{e}+P_{J}\right) / C_{p}
$$

where $C_{p}$ is the heat capacity of the battery. At $\mathrm{SOC}_{1}$ during discharge $-P_{e}=P_{J}$. At SOC $>\mathrm{SOC}_{1}$ during discharge $P_{e}+P_{J}<0$ and the battery cools down. At SOC $<\mathrm{SOC}_{1}$ during discharge $P_{e}+P_{J}>0$ and the battery heats up. At $\mathrm{SOC}_{3}$ during charge $-P_{e}=P_{J}$. At SOC $<\mathrm{SOC}_{3}$ during charge $P_{e}+P_{J}<0$ and the battery cools down. At SOC $>\mathrm{SOC}_{3}$ during discharge $P_{e}+P_{J}>0$ and the battery heats up.

The module voltage presented in Figure 2 was used to calculate the voltage difference between charging and discharging as shown by the gray points in Figure 3. Here two local maxima for the voltage difference are observed. One at about $25 \%$ SOC and one at about $75 \%$ SOC. The voltage difference increases with increasing resistance. The local maximum of the voltage difference at app. 25\% SOC occurs when the battery module temperature reaches a local minimum. Such a temperature dependence of the internal resistance is also expected and can be explained by the thermally activated electrode reactions. ${ }^{14}$ However, the local maximum of the voltage difference at app $75 \%$ SOC occurs when the battery temperature reaches a local maximum. This means the two local minima in the voltage difference cannot be explained in a simple manner by thermal activation.

The next part of the discussion section examines the impedance measurements and provides an expression for the internal resistance of the battery.

As stated above, the Single-Sine method is the most accurate method at high frequency but slow at low frequencies compared to the Laplace technique. With the single sine-technique it takes approximately 30 hours to measure impedance spectra on 8 cells plus the module down to $0.6 \mathrm{mHz}$. With the Laplace technique it only takes one hour. The remaining data from $60 \mathrm{mHz}$ to $2610 \mathrm{~Hz}$ measured with the single sine method on 8 cells plus the module takes less than 30 minutes. For this reason a much faster data acquisition can be obtained together with a good precision in the entire frequency range when the two measurement techniques are combined. Thus, in the current example it is possible to reduce the data acquisition time with a factor of 20 compared to ordinary single-sine measurements.

Equation 3 describes how the battery voltage evolves with time due to a step current. If the amplitude of the step current is sufficiently small and the battery is charged or discharged for a sufficiently long time we can neglect the transients and ignore changes in the impedance due to changes in SOC. Then equation 3 reduces to

$$
U_{m}(t)=u(t) \cdot I_{0}\left[R_{s}+t \cdot C_{b a t}+R_{1}+R_{2}+R_{3}+R_{4}\right]
$$

This means the voltage difference, $\Delta U$, between the battery voltage measured at the same SOC during charging and discharging can be obtained from equation 10 as

$$
\Delta U=U_{m}^{c}(t)-U_{m}^{d}(t)=2 I_{0}\left[R_{s}+R_{1}+R_{2}+R_{3}+R_{4}\right]
$$

where $U_{m}^{c}(t)$ and $U_{m}^{d}(t)$ is the battery voltage measured at the same SOC during charging and discharging, respectively. Thus the internal resistance as a function of SOC can be measured from a very slow constant-current charge-discharge curve as $\Delta U / 2 I_{0}$.

In relation to equation 3 , the number of (RC)-circuits was chosen to be the smallest possible number that provides a good fit between the measured overvoltage and the modeled overvoltage. A generic expression for the internal resistance of the battery, $R_{i}$, would be

$$
R_{i}=R_{s}+\sum_{n=1}^{N} R_{n}
$$

where $N$ is the number of (RC)-circuits. 

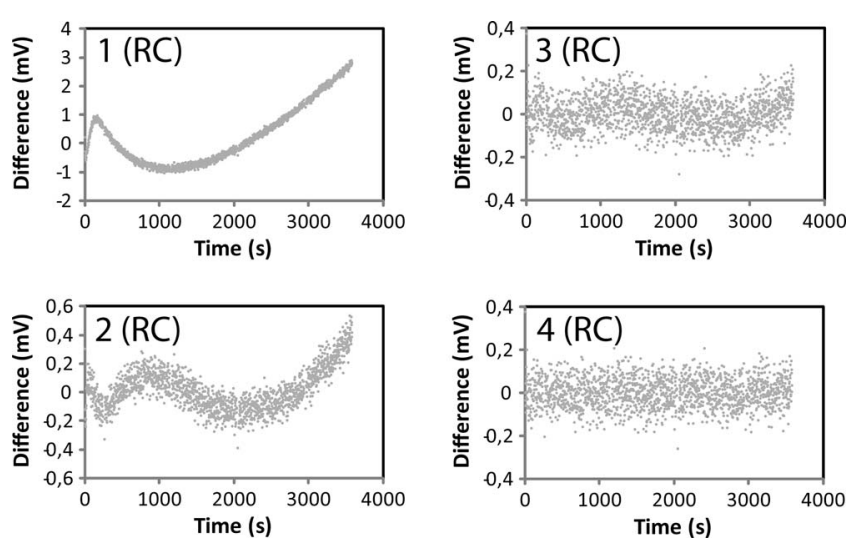

Figure 8. The residual between the measured and calculated overvoltage shown in Figure 4 using an expression similar to equation 3 but with a varying number of (RC)-circuits. The 4 (RC) figure is similar to the inset in Figure 4. The corresponding impedance spectra are shown in Figure 5.

We have modeled the overvoltage in Figure 4 with an expression like the one presented in equation 3 but with 1,2,3 and 4 (RC)s, i.e. $N=1,2,3$ and 4. Naturally, the difference between the measured and calculated overvoltage decreases with $N$ as seen from Figure 8 . In agreement with this, the single-sine measurements and Laplacemethod measurements increasingly overlap with increasing number of (RC)-circuits, as seen from Figure 5.

Ideally, the internal resistance given by equation 12 should convert asymptotically to a given value for an increasing number of (RC)circuits in the model. The obtained values of $R_{i}$ from the modeling are shown in Table I. Also the smallest characteristic frequency of the model (RC)-circuits are shown in the Table. The overvoltage measurement was conducted for one hour corresponding to a characteristic frequency of $0.28 \mathrm{mHz}$. The tabulated values for the characteristic frequency are comparable to, or even smaller than this frequency.

An exact determination of $R_{i}$ requires that the overvoltage measurement time is significantly longer than the characteristic time of the transients modeled by the (RC)s. If this had been the case, the inverse of $\omega_{0}$ would have been smaller than the measurement time. At the same time, the step current that causes the overvoltage needs to be sufficiently small to ensure a stable measurement of the battery impedance, i.e. that changes in the battery impedance due to the change in SOC during the overvoltage measurement can be ignored.

In the presented example, at least the first of these requirements were not fully satisfied and this explains why $R_{i}$ does not convert. However, the presented example demonstrates the principles of how $R_{i}$ can be measured.

The last part of the discussion section compares the voltage differences presented in Figure 3 with a calculated voltage difference derived from the measured impedance.

If we use the impedance obtained from the fit of the overvoltage in Figure 4 we can calculate how the battery voltage will evolve due to a series of 5 charge-steps of 6 minutes followed by 5 discharge steps of 6 minutes. In each charge/discharge step the battery is charged/discharged at $10 \mathrm{~A}$ for five minutes followed by one minute

Table I. Internal resistance and lowest characteristic frequency as a function of the number of (RC)-circuits.

\begin{tabular}{lcc} 
Number of (RC)-circuits & $R_{i}(\mathrm{~m} \Omega)$ & $\omega_{0}(\mathrm{mHz})$ \\
\hline 1 & 12,3 & 2,5 \\
2 & 15,9 & 0,31 \\
3 & 17,7 & 0,18 \\
4 & 19,4 & 0,13
\end{tabular}

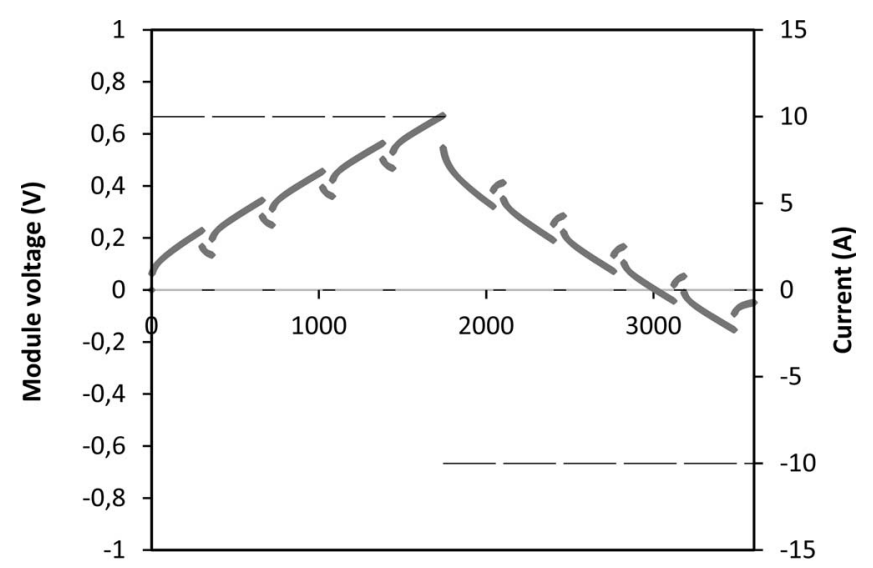

Time (seconds)

Figure 9. Calculated overvoltage and current as a function of time for the 75 Ah Kokam module due to a series of charge-discharge steps.

at $0 \mathrm{~A}$. Thus the total duration of the series of charge and discharge steps is 60 minutes, i.e. the same as the measurement time in Figure 4.

The current during the series of charge-discharge steps can be described by equation 13

$$
\begin{aligned}
I(t)= & I_{0} \sum_{n=0}^{4} u(t-n \cdot 6 \min )-u(t-n \cdot 6 \min -5 \min ) \\
& -u(t-(n+5) \cdot 6 \min )+u(t-(n+5) \cdot 6 \min -5 \min )
\end{aligned}
$$

$t$ is the time and $I_{0}=10 \mathrm{~A}$. Then, using $U(s)=Z(s) I(s)$, where $U(s)$, $Z(s)$ and $I(s)$ are the Laplace transformed voltage, impedance and current we can calculate the overvoltage $U(t)$ as a function of time. $I(t)$ and $U(t)$ are shown in Figure 9.

Using the nominal capacity of the battery module and assuming the impedance of the battery module is relatively independent of SOC between 88 and $94 \%$ SOC we can calculate the overvoltage as a function of SOC as shown in Figure 10. As seen from the figure, we can measure the voltage difference just before onset of the oneminute waiting periods at $0 \mathrm{~A}$ at four different SOCs. These voltage differences are also shown in Figure 10.

At $90 \%$ SOC, the calculated voltage difference in Figure 10 is $0.272 \mathrm{~V}$. This is in reasonable agreement with the measured voltage

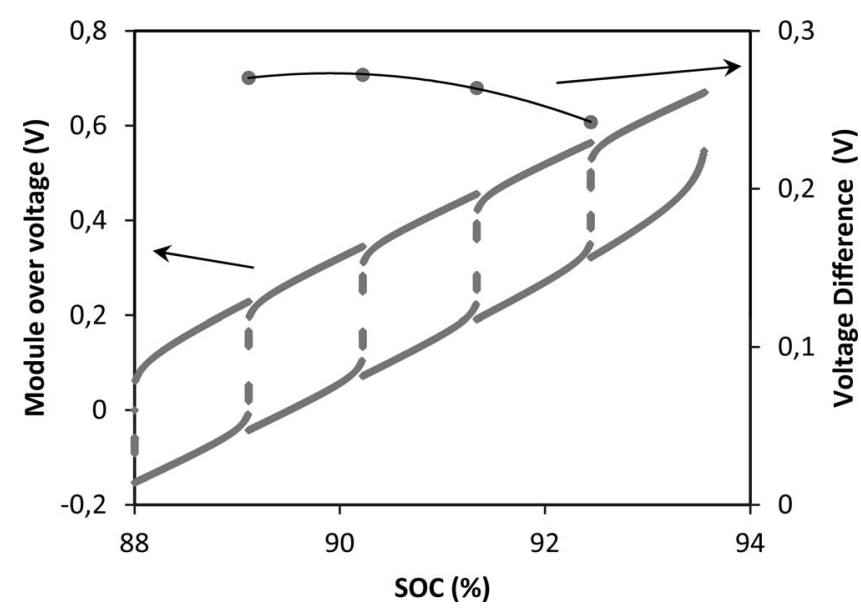

Figure 10. Calculated overvoltage and voltage difference as a function of SOC. 
difference at $90 \%$ SOC which is $0.322 \mathrm{~V}$. The difference in the calculated and measured voltage difference is believed to be due to the limited time between the charge- and discharge-measurements for the calculated voltage difference compared to the time between chargeand discharge- voltage measurements for the voltage difference measurements.

Another reason for the difference could be that the implicit linearization between overvoltage and current that is used for impedance measurements are satisfied for the measured voltage differences. However, this is thought to be a minor error: In the Kokam module the overvoltage at $90 \%$ SOC on the individual cells are $0.322 \mathrm{~V} /(2 \cdot 8)$ $=20 \mathrm{mV}$. The redox reactions involving $\mathrm{Li}^{+}$are normally singleelectron processes which means the error due to linearization of e.g. the Buttler-Volmer equation is less than $1 \%$.

\section{Conclusions}

The voltage and temperature were measured during a full chargedischarge cycle on a 75 Ah NMC battery module supplied from KOKAM. The module temperature exhibited a complex behavior during the charge-discharge cycle which is most likely due to the structural transformations and phase changes in the battery electrodes during the changes in the state of charge of the electrodes. The internal resistance of the module was derived from the voltage measured during the charge-discharge cycle. The internal resistance exhibits two local maxima at app. $25 \%$ SOC and $75 \%$ SOC. The local maximum of the internal resistance at $25 \%$ SOC correlates with a local minimum of the battery module temperature. This can be explained by the thermally activated electrochemical reactions in the battery electrodes. The local maximum of the resistance at $75 \%$ SOC occurs at a local maximum of the module temperature and is not fully understood.

The battery module impedance as well as the impedance of a single cell in the module were measured both with single-sine measurements and with a Laplace transformed step-current voltage response, referred to as a time domain measurement (TDM). The two techniques show good correlation at frequencies below $60 \mathrm{mHz}$ but deviate at higher frequencies. The TDM measurements can be conducted simultaneously on both the battery module and all the cells in the module whereas the single sine measurement technique is conducted on either the module or one of the cells at a time. For this reason it is possible to reduce the impedance measurement time on the NMC module and all its cells.
In the current example it is possible to reduce the measurement time with a factor of 20 compared to ordinary single-sine measurements.

The difference between the module voltage during charge and discharge was measured. From the measured impedance we calculate a voltage difference and show that it is in reasonable agreement with the measured voltage difference.

\section{Acknowledgments}

The authors thank Torben Jacobsen, ass. prof. emeritus, Dept. of Chem., Technical University of Denmark who suggested the presented method to convert TDM measurements into the frequency domain. Further we thank the Edison project funded by the ForskEl program (ForskEL Project Number 081216) and the Program Commission on Sustainable Energy and Environment, The Danish Council for Strategic Research, via the Strategic Electrochemistry Research Center (www.serc.dk), contract no. 2104-06-0011 for financial support to this work. Further, we thank Ris $\varnothing$ DTU, ABF for technical assistance in construction of the test setup and interest in this work.

\section{References}

1. M. Safari, M. Morcrette, C. Delacourt, M. Safari, and A. Teyssot, J. Electrochem. Soc., 157, A892 (2010)

2. U. Tröltzsch, O. Kanoun, and H. R. Tränkler, Electrochim Acta, 51, 1664 (2006).

3. J. Vetter, P. Novák, M. R. Wagner, C. Veit, K. C. Möller, J. O. Besenhard, M. Winter, M. Wohlfahrt-Mehrens, C. Vogler, and A. Hammouche, J. Power Sources, 147, 269 (2005).

4. E. Barsoukov, S. H. Ryu, and H. Lee, J. Electroanalytical Chem., 536, 109 (2002).

5. Y. Van Ingelgem, E. Tourwé, J. Vereecken, and A. Hubin, Electrochim Acta, 53, 7523 (2008).

6. Y. Van Ingelgem, E. Tourwé, O. Blajiev, R. Pintelon, and A. Hubin, Electroanalysis, 21, 730 (2009).

7. B. A. Boukamp, Solid State Ionics, 169, 65 (2004).

8. K. Onda, M. Nakayama, K. Fukuda, K. Wakahara, and T. Araki, J. Electrochem. Soc. (USA), 153, A1012 (2006).

9. D. Klotz, M. Schönleber, J. P. Schmidt, and E. Ivers-Tiffee, Electrochim Acta, 56, 8763 (2011).

10. S. Abu-Sharkh and D. Doerffel, J. Power Sources, 130, 266 (2004).

11. R. E. Williford, V. V. Viswanathan, and J. G. Zhang, J. Power Sources, 189, 101 (2009).

12. S. Al Hallaj, R. Venkatachalapathy, J. Prakash, and J. R. Selman, J. Electrochem. Soc., 147, 2432 (2000)

13. S. Al Hallaj, J. Prakash, and J. R. Selman, J. Power Sources, 87, 186 (2000).

14. N. Sato, J. Power Sources, 99, 70 (2001) 